\title{
Análises parasitológicas de hortaliças comercializadas na feira livre do Ver-o-Peso, Belém - PA
}

\author{
Parasitological analyses of vegetables commercialized at the free fair of Ver-o-Peso, \\ Belém - PA
}

Analizaciones parasitologicas de vegetables comercializados en la feria gratuita de Ver-oPeso, Belém - PA

Amanda Brito do Nascimento ${ }^{1,2 *}$, Suzana Ribeiro de Melo Oliveira ${ }^{1}$; Elem Cristina Rodrigues Chaves $^{3}$, Sergio Beltrão de Andrade Lima ${ }^{4}$, Tinara Leila de Souza Aarão ${ }^{4}$, Maria Helena Rodrigues de Mendonça $a^{4,2}$.

\section{RESUMO}

Objetivo: Avaliar a ocorrência de diferentes formas parasitárias em amostras de Lactuca sativa, Brassica oleracea, Coriandrum sativum, Petroselinum crispum, Allium schoenoprasum, Ocimum basilicum, Ocimum pilosum, Cichorium endivia e Mentha spicata provenientes do mercado Ver-o-Peso. Métodos: Estudo transversal, descritivo e quantitativo. Foram coletadas 5 amostras de cada hortaliça, sendo processadas pelos métodos de sedimentação espontânea e centrifugação, seguida de análise e identificação de parasitas. Resultados: Todas as amostras estavam contaminadas por parasitas. A hortaliça Lactuca sativa apresentou a maior frequência, entre os helmintos (82,24\%), os ovos de Ancylostoma sp e Ascaris sp., destacaram-se em ambos os métodos, entretanto, nos protozoários (60,05\%), o Balantidium sp (cistos) foi predominante no método de Hoffman e, no de Ritchie, foram os cistos de Entamoeba sp e Larvas de Nematodos. Comparando a detecção entre métodos, não houve diferença significativa, entretanto, ressalta importância das duas metodologias concomitantes para detecção de parasitas. Conclusão: A presença de parasitas em hortaliças significa risco na sua transmissão, além de criar um alerta quanto à provável subnotificação de parasitoses intestinais. A conscientização da correta higienização de alimentos é fundamental para redução das parasitoses, devendo ser acompanhada da efetiva atuação da vigilância sanitária nas feiras livres.

Palavras-chave: Hortaliças, Feiras livres, Parasitas intestinais.

\section{ABSTRACT}

Objective: To evaluate the occurrence of different parasitic forms in samples of Lactuca sativa, Brassica oleracea, Coriandrum sativum, Petroselinum crispum, Allium schoenoprasum, Ocimum basilicum, Ocimum pilosum, Cichorium ethyl and Mentha spicata from the Ver-o-Peso market. Methods: Cross-sectional, descriptive and quantitative study. Fifty samples of each vegetable were collected, being processed by spontaneous sedimentation and centrifugation methods, followed by analysis and identification of parasites. Results: All samples were contaminated by parasites. The Lactuca sativa vegetable showed the highest frequency among helminths (82.24\%), eggs of Ancylostoma sp and Ascaris sp., stood out in both methods,

\footnotetext{
${ }^{1}$ Faculdade Integrada Brasil Amazônia (FIBRA), Belém - PA. *E-mail: amanda.niely@hotmail.com

2 Instituto Evandro Chagas (IEC), Ananindeua - PA.

${ }^{3}$ Faculdade Integrada Brasil Amazônia- FIBRA, Belém - PA.

${ }^{4}$ Centro Universitário Metropolitano da Amazônia - UNIFAMAZ, Belém - PA.
}

SUBMETIDO EM: 10/2019

ACEITO EM: 11/2019

PUBLICADO EM: 2/2020 
however, in protozoan (60.05\%), Balantidium sp (cysts) was predominant in the method of Hoffman and, in Ritchie's, it was the cysts of Entamoeba $s p$ and Nematodos Larvae. Comparing the detection between methods, there was no significant difference, however, it emphasizes the importance of the two concomitant methodologies for the detection of parasites. Conclusion: The presence of parasites in vegetables means a risk in their transmission, in addition to creating an alert regarding the probable underreporting of intestinal parasites. Awareness of the correct hygiene of food is fundamental for reducing parasitics, and should be accompanied by the effective performance of health surveillance in free fairs.

Keywords: Vegetables, Free fairs, Intestinal parasites.

\section{RESUMEN}

Objetivo: Evaluar la aparición de diferentes formas parasitarias en muestras de Lactuca sativa, Brassica oleracea, Coriandrum sativum, Petroselinum crispum, Allium schoenoprasum, Ocimum basilicum, Ocimum pilosum, Cichorium ethyl y Mentha spicata del mercado Ver-o-Peso. Métodos: Estudio transversal, descriptivo y cuantitativo. Se recogieron cincuenta muestras de cada vegetal, siendo procesadas por métodos espontáneos de sedimentación y centrifugación, seguidos del análisis y la identificación de parásitos. Resultados: Todas las muestras fueron contaminadas por parásitos. El vegetal Lactuca sativa mostró la mayor frecuencia entre los helmintos (82,24\%), los huevos de Ancylostoma sp y Ascaris sp., destacaron en ambos métodos, sin embargo, en protozoario $(60,05 \%)$, Balantidium $s p$ (quistes) fue predominante en el método de Hoffman y, en Ritchie's, fueron los quistes de Entamoeba sp y Nematodos Larvas. Comparando la detección entre métodos, no hubo diferencia significativa, sin embargo, hace hincapié en la importancia de las dos metodologías concomitantes para la detección de parásitos. Conclusión: La presencia de parásitos en las verduras significa un riesgo en su transmisión, además de crear una alerta con respecto a la probable subinformación de los parásitos intestinales. El conocimiento de la correcta higiene de los alimentos es fundamental para reducir los parásitos y debe ir acompañado de la realización efectiva de la vigilancia sanitaria en las ferias libres.

Palabras clave: Vegetales, Ferias gratuitas, Parásitos intestinales

\section{INTRODUÇÃO}

Em países recentemente industrializados com economias emergentes, como o Brasil, os endoparasitos intestinais são comumente propagados tanto no meio rural quanto em áreas urbanas. O favorecimento ao acometimento de pessoas por enfermidades intestinais se dá pelo fato do país ter um clima e situação socioeconômica favorável (MESQUITA VCC, et al., 1999). A má higienização dos alimentos consumidos in natura tem sido apontada como um fator favorável à disseminação das doenças parasitárias, tornando-se um grave problema de saúde pública no Brasil (MESQUITA VCC, et al., 1999; SILVA CGM, et al., 2005; FREI F, et al., 2008)

As parasitoses intestinais ou enteroparasitoses são apontadas como um indicador do desenvolvimento socioeconômico de um país, a alta prevalência de protozoários e/ou helmintos afeta mais de $30 \%$ da população mundial, principalmente, nos países em desenvolvimento, decorrente das más condições de vida, precárias condições de saneamento básico, baixo nível socioeconômico e a falta de orientação educacional, demonstrando o pouco investimento na promoção e prevenção em saúde pelos governos locais, principalmente nas camadas populacionais mais carentes (FREI F, et al., 2008; MACIEL DF, et al., 2014; SILVA AO, et al., 2015).

O Brasil, como um país tropical, possui clima favorável para produção de hortaliças, sendo a agricultura familiar responsável por mais da metade da produção, as formas parasitárias de protozoários e helmintos podem contaminar esses alimentos durante o cultivo, por meio do uso de esterco animal, água de irrigação sem tratamento adequado, além de transporte e armazenamento inadequados destinados ao consumo humano in natura (FREI F, et al., 2008; FERNANDES NS, et al., 2015). 
As hortaliças estão presentes na dieta diária devido ao teor de nutrientes necessários ao funcionamento adequado do organismo, como sais minerais, fibras alimentares e vitaminas, além de apresentarem ação antioxidante, esses atributos têm aumentado o consumo, principalmente pelos que buscam um estilo de vida mais saudável (CARVALHO PGB, et al, 2006).

Entretanto, já foi demonstrado que tais tipos de alimentos possuem alta probabilidade de apresentar ovos e larvas de helmintos, assim como cistos de protozoários derivadas de águas contaminadas por dejetos fecais de animais e/ou homem, sendo considerada, uma importante fonte de transmissão que precisa ser monitorada (COELHO LMPS, et al., 2001; SILVA CGM, et al., 2005; CAPUANO DM, et al., 2008; FERNANDES NS, et al., 2015).

O estilo de vida saudável adotado pela população faz com que haja o aumente no consumo de hortaliças devido a essa alteração no padrão de vida. Hortaliças como a couve (Brassica sylvestris), hortelã (Mentha spicata) e chicória (Cichorium intybus) são muito utilizadas devido as suas propriedades medicinais que são compostas por vitaminas, fibras alimentares e sais minerais que auxiliam a saúde (PINEZI JMI e ASCHIDAMINI IM, 2011; SIMOES RB, et al.,2016).

Outro fato importante é o uso de temperos na comida, com o intuído de dar mais vida e sabor aos pratos, a culinária brasileira é marcada pelos seus sabores únicos. Por isso, utiliza uma extensa variedade de temperos que acrescentam riqueza e requinte aos pratos. A região Norte é marcada por utilizar muito Coriandrum sativum (coentro), Ocimum basilicum (Manjericão) e Petroselinum (Salsa). Essas especiarias podem trazer benefícios à saúde devido aos seus nutrientes e ajudar na conservação dos alimentos (ZANUSSO GJ, et al., 2011; FRANZ DW, et al., 2015).

Na cidade de Belém, no Estado do Pará, encontramos o Mercado do Ver-o-Peso, a maior feira ao ar livre da América Latina, está é reconhecida como patrimônio histórico pelo Instituto do Patrimônio Histórico e Artístico Nacional (Iphan). O Ver-o-Peso vende de tudo, desde peixes, carnes, frutas e legumes até artigos regionais, ervas medicinais, plantas ornamentais e raízes aromáticas, recebe cerca de 50 mil pessoas diariamente. O fluxo intenso, aliado ao comercio e manipulação das hortaliças folhosas oferece facilidade para possível contaminação (BARBOSA V, 2009).

Visando a importância do consumo de hortaliças, como fontes de vitaminas, fibras, nutrientes, antioxidantes e carboidratos para população, assim como, a possibilidade de contágio por enteroparasitas por meio destes alimentos e ainda considerando a alta incidência e prevalência das doenças parasitárias intestinais, cujos agentes são eliminados nas fezes humanas, impõe-se a necessidade de adoção de medidas para monitorar e incentivar a adoção de boas práticas na manipulação destes alimentos a fim de minimizar possíveis infecções por enteroparasitas (COELHO LMPS, et al., 2001; FILHO JCR, 2016)

Objetivo: Avaliar a ocorrência de diferentes formas parasitárias em amostras de hortaliças folhosas provenientes da feira livre do Ver-O-Peso da cidade de Belém - PA.

\section{MÉTODOS}

Consiste em um estudo transversal, observacional e quantitativo. Onde foram adquiridas 5 amostras de cada hortaliça em barracas aleatórias da feira livre do Ver o Peso da cidade de Belém/PA. O tratamento das hortaliças foi baseado nos métodos de sedimentação espontânea e centrifugação (Hoffman e Ritchie adaptado) respectivamente, seguido da análise e identificação dos parasitas. Foram confeccionadas e lidas 2250 lâminas, sendo 250 lâminas para cada hortaliça (125 por método). Para a construção dos gráficos usouse o programa GraphPad Prism $6{ }^{\circledR}$ para a avaliação das médias, frequências e comparação dos percentuais de contaminação foi usado o programa BioEstat 5.0 (AYRES M, et al., 2007).

\section{Amostras}

Foram coletadas 5 amostras de cada hortaliça: Alface (Lactuca sativa), Couve (Brassica sylvestris), Chicória (Cichorium intybus) e Hortelã (Mentha spicata), Coentro (Coriandrum sativum), Manjericão (Ocimum 
basilicum), Salsa (Petroselinum), Cebolinha (Allium schoenoprasum) e Alfavaca (Ocimum campechianum) na feira livre do Ver-o-Peso (Mercado municipal) no período de Janeiro a Junho de 2017. Para a coleta do material, foram utilizados sacos plásticos para transporte ao laboratório e fichas de registros para anotações da procedência, espécie de verdura e data.

As hortaliças foram desfolhadas, fatiadas e homogeneizadas. Foi adicionado o Tampão Phosphatebufferid saline (PBS) $(8,5 \mathrm{~g}$ de Cloreto de Sódio-NaCl; 1,55g de Fosfato dissódico-Na2HPO4. 2H2O; 0,23g de Fosfato Sódio Monobásico -NaH2PO4.H2O ; pH 7,2 - 7,4, concentrado10x e diluído em 900ml de água destilada. Foi adicionado $500 \mathrm{~mL}$ de tampão e homogeneizado com bastão de vidro por 20 minutos e retirado o excesso de folhas do líquido.

\section{Pesquisa de cistos de protozoários, ovos e larvas de helmintos: sedimentação espontânea (Hoffman e Ritchie adaptado).}

Para a filtragem do líquido resultante da mistura das hortaliças, foi utilizado dois cálices de sedimentação e uma gaze dobrada em quatro foi colocado $200 \mathrm{~mL}$ em cada cálice e o restante foi distribuído em dois tubos cônicos de $50 \mathrm{~mL}$. Para o método de Hoffman, foi deixado em repouso por 24 horas. Após esse período, com uma pipeta Pasteur, foi retirada uma amostra do fundo do vértice do cálice e foi colocado em lâmina uma gota do material sedimentado e uma gota de lugol 1\%, as laminas foram examinadas ao microscópio com o aumento de 10x e, ao identificar algum enteroparasita, utilizou a de 40x para a confirmação.

Para o método de Ritchie adaptado, o material foi depositado em tubos cônicos de $50 \mathrm{~mL}$ e centrifugado a 3000 rpm por 3 minutos, o sobrenadante foi desprezado. Com uma pipeta Pasteur foi retirada uma amostra do fundo do vértice do cálice, e colocada em lâmina uma gota do material sedimentado e uma gota de lugol $1 \%$, as laminas foram examinadas ao microscópio, com o aumento de $10 \mathrm{X}$ e, ao identificar algum enteroparasita, de 40X para a confirmação.

\section{RESULTADOS E DISCUSSÃO}

Neste trabalho, foram analisadas 9 hortaliças (Alface, couve, coentro, salsa, cebolinha, alfavaca, manjericão, chicória e hortelã) comercializadas na feira livre do Ver-o-Peso, cidade de Belém, que são normalmente consumidas pela população local, todas as amostras estavam contaminadas por alguns organismos protozoários, helmintos e/ou larvas de nematodos, como está demonstrado na (Tabela 1).

A alface (Lactuca sativa) apresentou a maior frequência de ovos de helminto tanto pelo método de Hoffman (77,92\%) quanto pelo Ritchie (80,54\%) e cistos de protozoários pelo Hoffman (45,95\%) e Ritchie (58,93\%), entretanto, o manjericão apresentou uma maior frequência de larvas de nematodos nos dois métodos, sendo Hoffman $55.67 \%$ e Ritchie $32.08 \%$.

A hortaliça que menos apresentou ovos e larvas foram às cebolinhas, não sendo encontrado nem uma forma de protozoário na mesma (Tabela 1). As médias de formas parasitárias detectadas nas cinco amostras de alface, tanto pelo método de Hoffman, assim como no método de Ritchie modificado é preocupante, destacase ovos de Ascaris sp., cistos de Entamoeba sp., trofozoítas de Balantidium sp e ovos de Ancylostoma sp (Figura 1A).

Para alfavaca (Ocimum campechianum) e a cebolinha (Allium schoenoprasum), o predomínio foi de larvas de nematodos (Figura 1B e C). Entretanto, a couve (Brassica sylvestris), apresentou mais cistos de Entamoebas $s p$. e trofozoítas de Balantidium sp. (Figura 2A), larvas de nematodos foram mais frequentes nas amostras de hortelã (Mentha spicata) e chicória (Cichorium intybus) (Figura 2B e C).

As amostras de coentro (Coriandrum sativum) e manjericão (Ocimum basilicum) apresentaram trofozoítas de Balantidium sp. (Figura 3A e B), mas as de salsa (Petroselinum) foram observadas mais larvas de nematodos (Figura $\mathbf{3 C}$ ). 


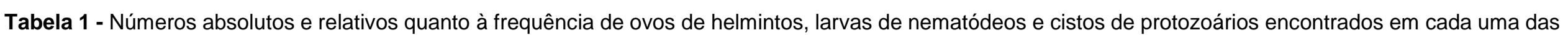
nove hortaliças comercializadas em uma feira livre da cidade de Belém - PA.

\begin{tabular}{|c|c|c|c|c|c|c|}
\hline & \multicolumn{2}{|c|}{ OVOS DE HELMINTOS (\%) } & \multicolumn{2}{|c|}{ LARVAS DE NEMATODOS (\%) } & \multicolumn{2}{|c|}{ CISTOS DE PROTOZOÁRIOS (\%) } \\
\hline & HOFFMAN & RITCHIE & HOFFMAN & RITCHIE & HOFFMAN & RITCHIE \\
\hline Alface & 77.92 (1885) & (1684) 80.54 & (132) 12.58 & (243) 22.86 & (1524) 45.95 & 58.93 \\
\hline Couve & (278) 11.49 & (191) 9.13 & (26) 2.48 & (35) 3.29 & (691) 20.83 & (743) 30.09 \\
\hline Coentro & (136) 5.62 & (129) 6.17 & (67) 6.39 & (105) 9.88 ) & (293) 8.83 & (150) 6.08 \\
\hline Salsa & (24) 0.99 & (38) 1.82 & (43) 4.10 & (99) 9,31 & (699) 21.07 & (75) 3.04 \\
\hline Cebolinha & (2) 0.08 & (4) 0.19 & (7) 0.67 & (11) 1.03 & (0) 0.00 & 0.00 \\
\hline Alfavaca & (10) 0.41 & (7) 0.33 & (37) 3.53 & (46) 4.33 & (10) 0.30 & (10) 0.41 \\
\hline Manjericão & (15) 0.62 & (12) 0.57 & (584) 55.67 & (341) 32.08 & (81) 2.44 & (12) 0.49 \\
\hline Chicória & (17) 0.70 & (11) 0.53 & (53) 5.05 & (95) 8.94 & (2) 0.06 & (4) 0.16 \\
\hline Hortelã & (52) 2.15 & (15) 0.72 & 9.53 & (88) 8.28 & (17) 0.51 & (20) 0.81 \\
\hline
\end{tabular}

Fonte: Nascimento AB, et al., 2019. 
Figura 1 - Médias das formas parasitárias encontradas nas hortaliças (A) alface (Lactuca sativa), (B) alfavaca (Ocimum campechianum) e a (C) cebolinha (Allium schoenoprasum).

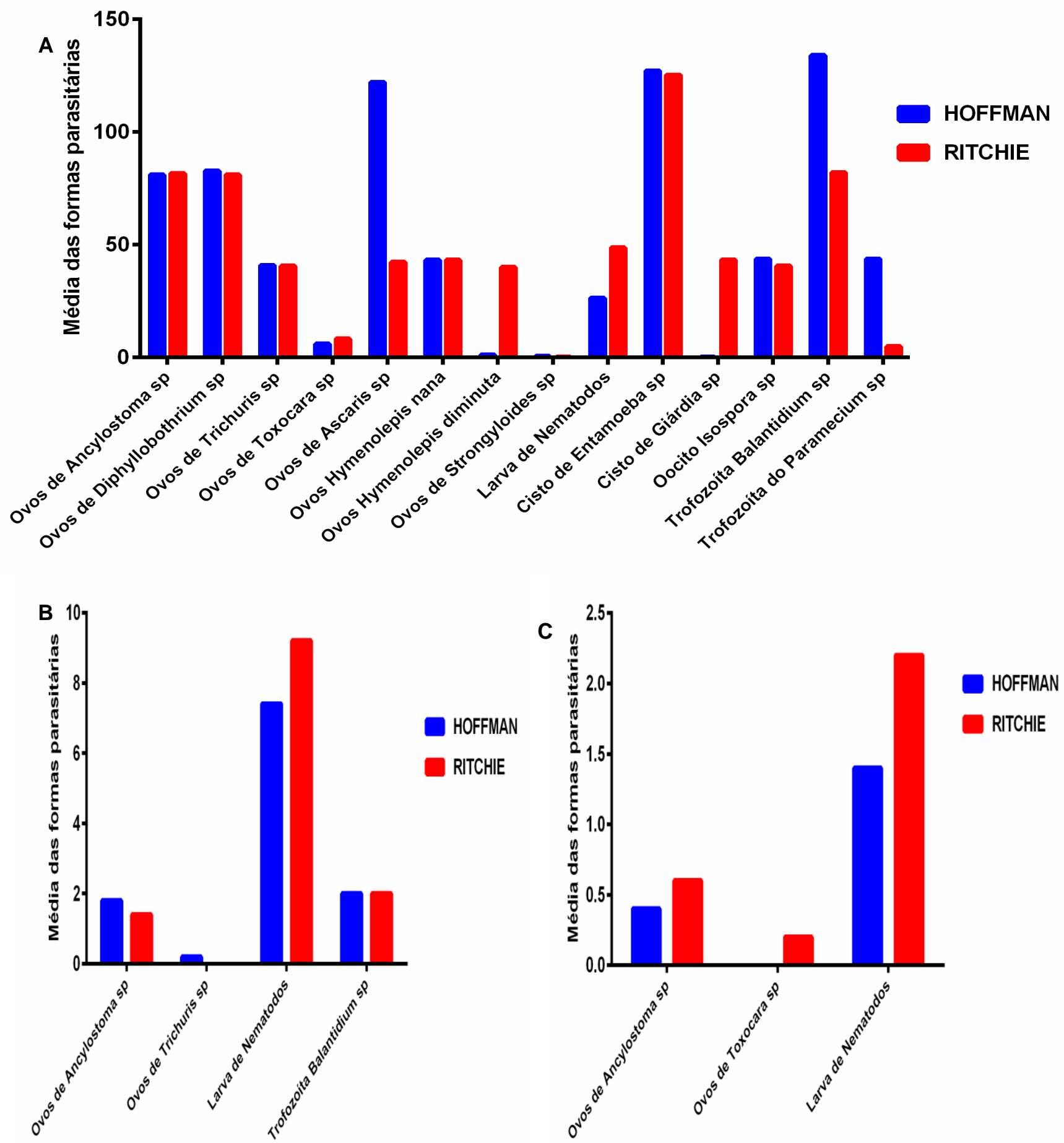

Legenda: Demonstra a média* de formas parasitárias encontradas em amostras de hortaliças comercializadas em uma feira livre da cidade de Belém - Pará, utilizando os métodos de Hoffman e Ritchie adaptados. A figura (A) é a alface (Lactuca sativa), (B) alfavaca (Ocimum campechianum) e a (C) cebolinha (Allium schoenoprasum).

*Médias das cinco amostras realizadas.

Fonte: Nascimento AB, et al., 2019. 
Figura 2 - Médias das formas parasitárias encontradas nas hortaliças (A) é a couve (Brassica sylvestris), (B) hortelã (Mentha spicata) e (C) chicória (Cichoriu mintybus).
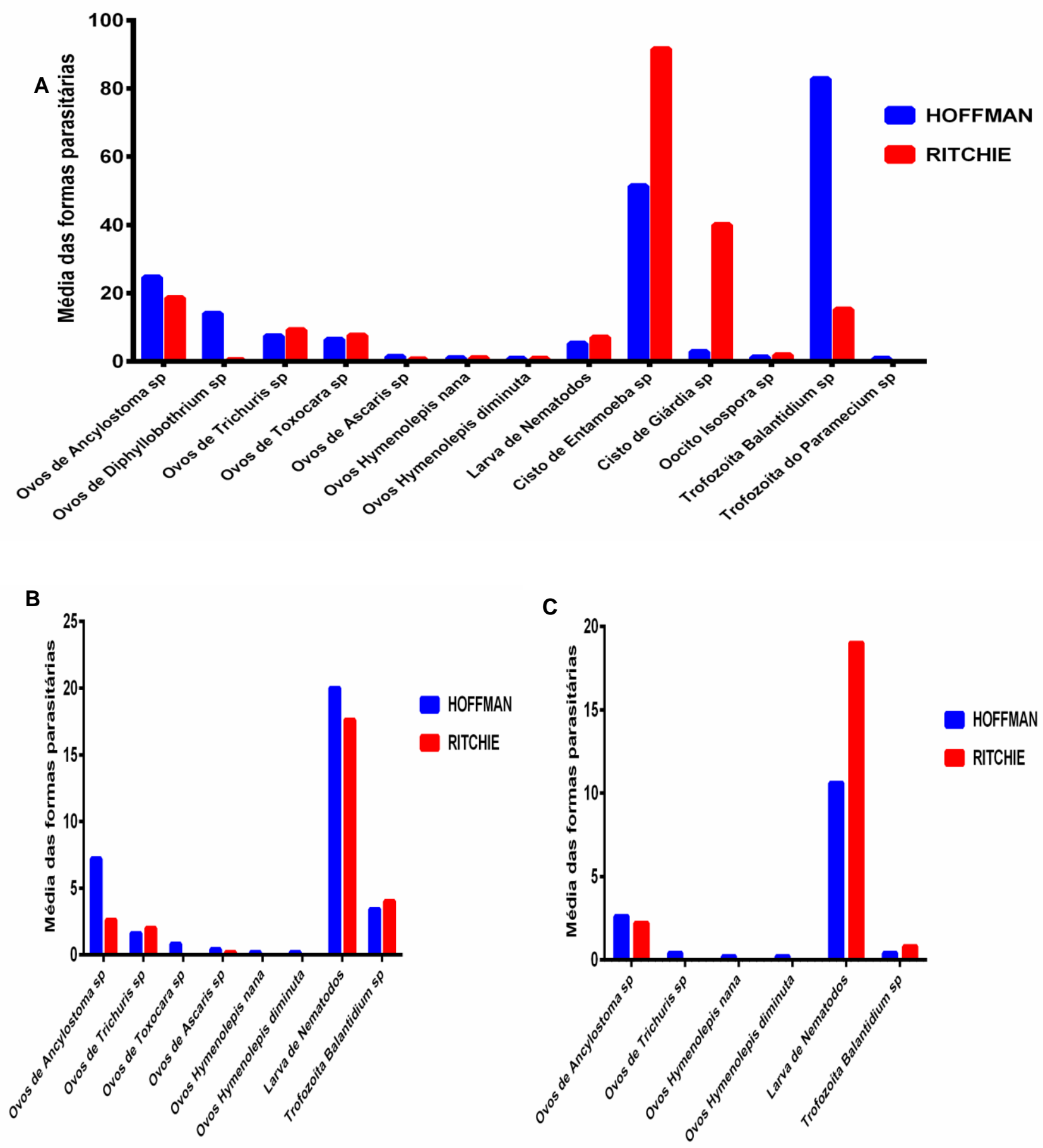

Legenda: Demonstra a média* de formas parasitárias encontradas em amostras de hortaliças comercializadas em uma feira livre da cidade de Belém - Pará, utilizando os métodos de Hoffman e Ritchie adaptados. A figura (A) é a couve (Brassica sylvestris), (B) hortelã (Mentha spicata) e (C) chicória (Cichoriu mintybus.

*Médias das cinco amostras realizadas.

Fonte: Nascimento AB, et al., 2019. 
Figura 3 - Médias das formas parasitárias encontradas nas hortaliças (A) é a coentro (Coriandrum sativum), (B) salsa (Petroselinum) e (C) manjericão (Ocimum basilicum).
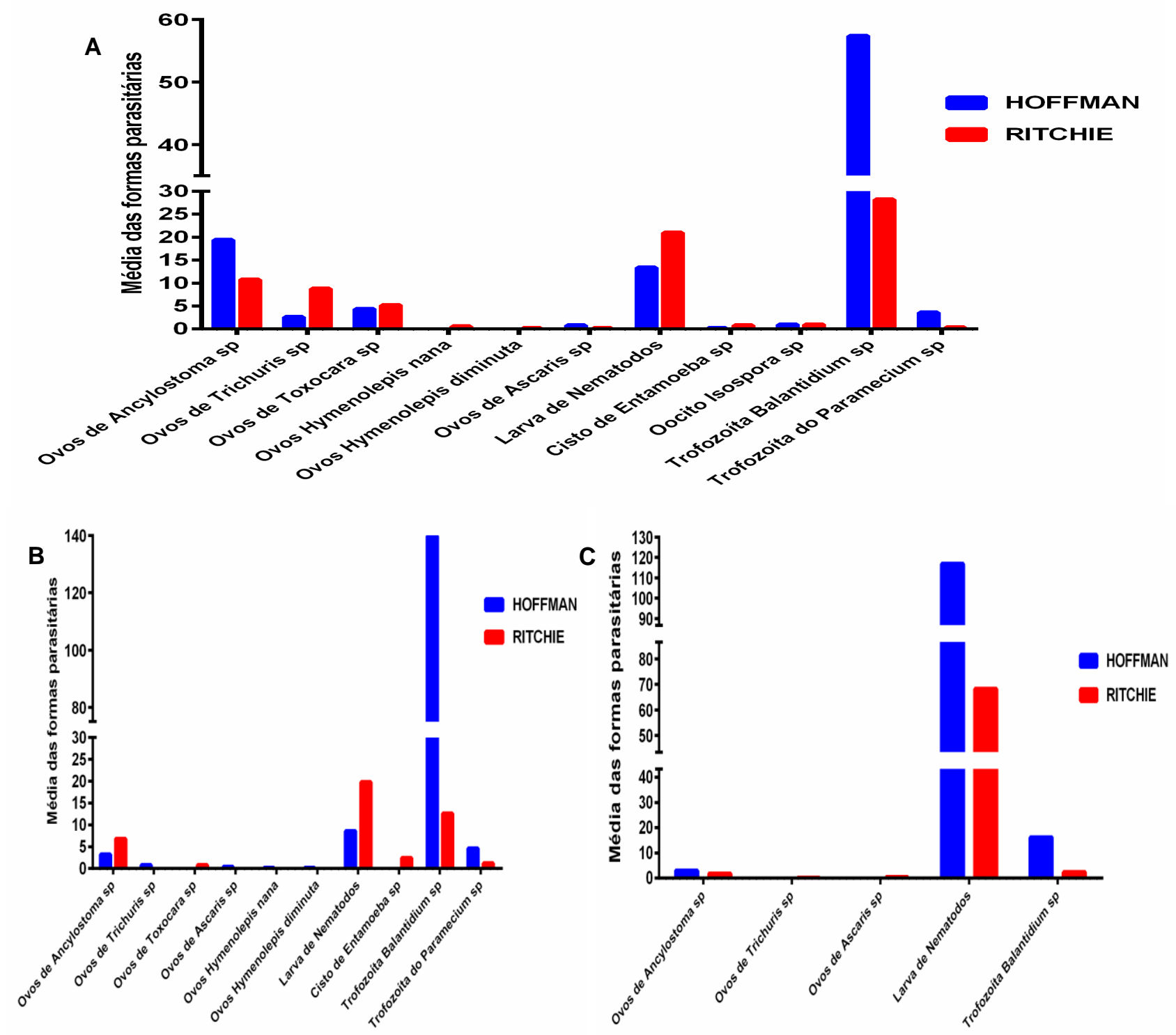

Legenda: Demonstra a média de formas parasitárias encontradas em amostras de hortaliças comercializadas em uma feira livre da cidade de Belém/Pará, utilizando os métodos de Hoffman e Ritchie adaptados. A figura (A) é a coentro (Coriandrum sativum), (B) salsa (Petroselinum) e (C) manjericão (Ocimum basilicum) * Médias das cinco amostras realizadas.

Fonte: Nascimento AB, et al., 2019.

Estas elevadas contaminações por endoparasitoses que foram encontrados nas nove hortaliças avaliadas por duas metodologias para a detecção de formas parasitárias, demonstra como é vulnerável as condições de higienes-sanitária da produção, transporte e comercialização dessas hortaliças. Leitão WM (2013), afirma que o abastecimento vegetal proveniente de ilhas próximas ao mercado Ver-O-Peso, localizado na região metropolitana de Belém, provêm diretamente da natureza, onde são aplicadas técnicas tradicionais, relativamente simples, fator que pode contribuir para presença de estruturas parasitárias, visto que este tipo de produção não disponibiliza condições e tecnologia adequadas de produção. 
De acordo com Pereira J (2010), condições precárias de saneamento nas áreas rurais e urbanas favorecem a transmissão de enteroparasitas presente em fezes animal e humano. Silva VG, et al. (2017) relatam elevada contaminação das hortaliças através de solo adubado por esterco animal, práticas inadequadas de irrigação e a higienização precária nas etapas de manipulação das hortaliças podem contribuir para grandes variedades de formas parasitárias encontradas. Quanto a baixa contaminação apresentada pela cebolinha, Oliveira DM, et al., (2016), relaciona a morfologia das folhas cilíndricas e simples dificultam a aderência de formas parasitárias. As folhas de alface e couve têm maior superfície de contato quanto comparada às folhas de cebolinha, o que facilita a adesão de microrganismos. Outros estudos apresentam resultados similares aos obtidos nessa pesquisa, o que significa que há perigo iminente de contaminação por parasitas, sendo um alerta para subnotificação de parasitoses intestinais prováveis, auxiliando à persistência de indivíduos portadores na região (LUZ J, et al., 2014; ALVES AS, et al., 2013; PIRES DR, et al., 2014).

A correta higienização dos alimentos pode reduzir as parasitoses, entretanto, deve-se fortalecer as campanhas de conscientização da população e uma melhor atuação da vigilância sanitária em feiras livres. Segundo Oliveira A e Perez L (2014), a contaminação das hortaliças não pode denegrir os benefícios proporcionados por esses alimentos quanto à presença de sais minerais e vitaminas, isso deve se sobressair à contaminação. Com isso, é necessária a conscientização da população que consome esses vegetais para que promovam a correta higienização para o consumo, pois, de acordo com Nascimento ED e Alencar FLS (2014), a higienização correta das hortaliças ocasiona a diminuição de amostras contaminadas por parasitas, além disso, relatam que utilização de produtos como hipoclorito de sódio e do ácido acético podem contribuir para diminuição de casos de doenças parasitárias transmitidas por alimentos.

A alta média de trofozoítas de Balantidium sp., observadas nas amostras, se deve à presença animal nas plantações, principalmente suínos, por serem um dos principais grupos de animais reservatório de balantidiose, de acordo com Barbosa AS (2015). Outro fato analisando, foi se haveria diferença na detecção de formas parasitárias por metodologias diferentes, entretanto, a detecção entre os métodos não apresentou diferença significativa ( $<0,05$ - Wilcoxon), contudo, é necessário que se ressalte a importância da realização das duas metodologias concomitantes para a detecção de formas parasitárias em hortaliças, isso traz mais segurança na deteç̧ão de ovos, larvas, cistos e trofozoítas de parasitas que podem contaminar os consumidores, assim sendo, mais uma contribuição em ações de vigilância sanitária e epidemiológica (Figura 4).

Figura 4 - Demonstra a comparação das metodologias Hoffman e Ritchie adaptados para constatação de formas de parasitas nas amostras de hortaliças vendidas em uma feira livre da cidade de Belém/Pará.

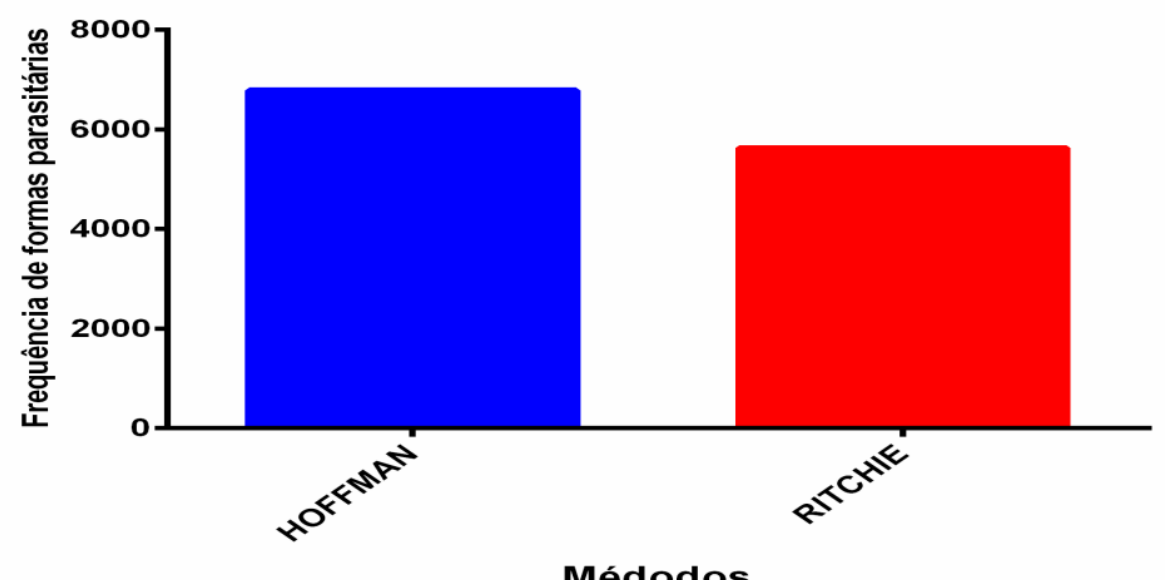

Legenda: Não foi observada diferença significativa entre as metodologias ( $<0,05$ - teste de Wilcoxon).

Fonte: Nascimento AB, et al., 2019;

REAS/EJCH | Vol.Sup.n.41 | e2135 |DOI: https://doi.org/10.25248/reas.e2135.2020 Página 9 de 11 


\section{CONCLUSÂO}

Esse estudo encontrou diferentes formas parasitárias nas hortaliças analisadas, provavelmente devido ao manuseio e manipulação dessas hortaliças durante plantio, transporte e comercialização. Com relação aos consumidores, a situação torna-se ainda mais crítica, quando os mesmos não são preocupados com a higienização das hortaliças. Os parasitas encontrados nas hortaliças vendidas em feiras livres como o mercado do Ver-o-Peso, apresenta risco de transmissão de parasitoses à população consumidora. Esse estudo expõe a importância de se aplicar programas de educação sanitária aos feirantes, com intuito de minimizar a contaminação de hortaliças comercializadas na no Ver-o-Peso e, também, realçar a necessidade de realizar a sanitização adequada das folhas antes do consumo, com objetivo de reduzir a ingestão dos endoparasitas.

\section{AGRADECIMENTOS}

Agradecemos a Beatriz Rocha Ferreira, Claudia Larissa Viana da Silva, Leonardo Henrique Almeida Hernández e Luiz Adriano Damasceno de Queiroz, discentes da professora Maria Helena Rodrigues de Mendonça, por participarem de parte do processo de elaboração do artigo, sendo seu resumo publicado em Anais do Evento Medtrop- 54 Congresso da Sociedade Brasileiro de Medicina Tropical no ano de 2018.

\section{REFERÊNCIAS}

1. ALVES AS, et al. Parasitos em alface-crespa (Lactuca sativa I.), de plantio convencional, comercializada em supermercados de Cuiabá, Mato Grosso, Brasil. Goiás. Revista de Patologia Tropical, 2013; v.42.

2. AYRES M, et al. BioEstat 5.0: Aplicações Estatísticas nas Áreas das Ciências Bio-médicas.5ํㅡㄹ Ed. Belém, Pará, 2007; 364p.

3. NASCIMENTO ED, ALENCAR FLS. Eficiência antimicrobiana e antiparasitária de desinfetantes na higienização de hortaliças na cidade de Natal - RN. Ciência e Natura, Santa Maria, v. 36 n. 2 mai-ago. 2014, p. 92-106

4. BARBOSA AS. Estudo de Balantidium sp. (Claparède e Lachmann, 1858) isolados de suínos, primatas não humanos cativos e humanos no Estado Rio do Janeiro, Brasil. Rio de Janeiro, RJ, 2015; p.251.

5. BARBOSA V Mercado Ver-o-Peso. Pesquisa Escolar Online, Fundação Joaquim Nabuco, Recife. Disponível em: <http://basilio.fundaj.gov.br/pesquisaescolar/>. Acesso em: 08 set. 2019.

6. CARVALHO PGB, et al. Hortaliças como alimentos funcionais. Hortic Bras. Vol. 24, Brasília, 2006.

7. CAPUANO DM, et al. Enteroparasitoses em manipuladores de alimentos do município de Ribeirão Preto - SP, Brasil, 2000. RevBrasEpidemio, 2008.

8. COELHO LMPS, et al. Detecção de formas transmissíveis de enteroparasitas na água e nas hortaliças consumidas em comunidades escolares de Sorocaba, São Paulo, Brasil. Revista da Sociedade brasileira de Medicina Tropical. $2001 ; 34$, pp. 479-482.

9. FERNANDES NS, et al. Avaliação parasitológica de hortaliças: da horta ao consumidor final. Revista Saúde e Pesquisa, 2015; V. 8, n. 2, p. 255-265.

10. FREI F, et al. Levantamento epidemiológico das parasitoses intestinais: viés analítico decorrente do tratamento profilático. Cad. Saúde Pública, Rio de Janeiro, 2008; 24(12):2919-2925.

11. FILHO JCR, BRASIL JB. Diagnóstico do consumo de hortaliças em uma instituição de ensino no nordeste brasileiro. Revista Ensino \& Pesquisa, 2016; v.14 n.01 p.136-144.

12. FRANZ DW, et al. Avaliação da salsa crespa (Petroselinum crispum) no sistema de horta vertical, 2015.

13. FREI $F$, et al. Levantamento epidemiológico das parasitoses intestinais: viés analítico decorrente do tratamento profilático. Cad. Saúde Pública, Rio de Janeiro, 2008; v. 24, n. 12, p. 2919-2925.

14. LEITÃO WM. VER-O-PESO: um mercado de coisas boas e belas. Uberlândia, MG, 2013.

15. LUZ J, et al. Avaliação da contaminação parasitária em alfaces (Lactuca sativa) comercializadas em feiras livres na região da Grande Natal, Rio Grande do Norte. Ceará. Nutrivisa, 2014; v. 1, Núm. 2 pág. 16-19.

16. MESQUITA VCC, et al. Contaminação por enteroparasitas em hortaliças comercializadas nas cidades de Niterói e Rio de Janeiro, Brasil. Rev. Soc. Bras. Med. Trop., 1999; v.34, n.4, p.189-194.

17. MACIEL DF, et al. Ocorrência de parasitos intestinais em hortaliças comercializadas em feiras no distrito federal, brasil. RevPatolTrop Vol. 43, 2014.

18. OLIVEIRA DM, et al. Perfil parasitológico do cheiro verde comercializado em feiras livres de Imperatriz-MA. Biota Amazônia (BioteAmazonie, Biota Amazonia, Amazonian Biota), 2016; v. 6, n. 2, p. 123-126. 
19. OLIVEIRA A, PEREZ L. Contaminação de enteroparasitas em folhas de alface (Lactuca sativa) e agrião (Nasturtiumofficinalis) em duas hortas comerciais de foz do Iguaçu, estado do Paraná, Brasil. Rio de Janeiro. Revista Eletrônica Novo Enfoque, 2014; v. 18, n. 18, p. 109 - 124.

20. PEREIRA JA. Avaliação da contaminação da alface (Lactuca sativa) variedade crespa por bactérias e enteroparasitas. Universidade Federal da Paraíba, João Pessoa, 2010.

21. PINEZI JMI, ASCHIDAMINI IM. REEDUCAÇÃO ALIMENTAR COMO MELHORIA DA QUALIDADE DE VIDA, Universidade Federal do Paraná-Litoral, 2011.

22. PIRES DR, et al. Avaliação parasitológica de alfaces (Lactuca sativa) comercializadas no município do Rio de Janeiro (RJ) Semina: Ciências Biológicas e da Saúde, Londrina, 2014; v. 35, n. 1, p. 35-48.

23. SILVA CGM, et al.. Ocorrência de Cryptosporidiumspp. E outros parasitas em hortaliças consumidas in natura, no Recife. Ciência \& Saúde Coletiva, 2005; v. 10, (sup). P. 63-69.

24. SILVA AO, et al. Epidemiologia e prevenção de parasitoses intestinais em crianças das creches municipais de itapuranga - GO. Revista Faculdade Montes Belos (FMB), 2015; v. 8, n 1.

25. SILVA VG, et al. Enteroparasitas veiculados em folhas de alfaces (Lactuca sativa) comercializadas na feira livre da cidade de Governador Valadares, Minas Gerais. Goiânia. ENCICLOPÉDIA BIOSFERA, Centro Científico Conhecer, 2017; v.14 n.25; p. 1343.

26. SIMOES RB, et al. Detox e sua real eficácia. Anais da 11ํ mostra de iniciação cientifica Junior, 2016.

27. ZANUSSO GJ, et al. Avaliação da atividade antiinflamatória do coentro (Coriandrumsativum L.) em roedores., Rev. Bras. Pl. Med., Botucatu, 2011; v.13, n.1, p.17-23. 